\title{
La Educación Inclusiva e Intercultural en la Práctica Educativa
}

\author{
Inclusive and Intercultural Education in Educational Practice
}

MARIANA DEL ROCÍO AGUILAR BOBADILLA

Universidad Pedagógica Nacional, Distrito Federal, Mexico (marianadelrocioa@outlook.com) (https://orcid.org/0000-0003-4427-3003)

\section{RESUMEN}

La concepción de la educación tiene implicaciones en la definición y gestión de la política educativa así como la propia dinámica de la institución escolar en la definición de sus procesos y la función de los actores educativos; en particular la responsabilidad social y política que recae en el docente. El propósito fue documentar y analizar la práctica docente propia con base en el acompañamiento en el desarrollo de los proyectos de investigación que sustentan las tesis, en el caso particular, de estudiantes de la Maestría en Educación Básica (MEB) con especialidad en Pedagogía de la Diferencia y la Interculturalidad en la Unidad 096 CDMX Norte de la Universidad Pedagógica Nacional. La asesoría es el acompañamiento en el desarrollo de los proyectos de investigación, considera la práctica docente propia como objeto de conocimiento y vínculo entre el trabajo académico con los procesos de aprendizaje en el aula; ésta se documentó en registros de observación. Para la comprensión del hecho educativo, en su fenomenología mediante una perspectiva interdisciplinar, permite recuperar la discusión que los docentes realizan, inicialmente, la conceptualización de las categorías de análisis que explican los fenómenos que vive la sociedad actual y tienen implicaciones en la escuela y, posteriormente, hacer la revisión y análisis de los planes de estudio, que son la base para la implementación de los mismos; en la orientación de la práctica docente propia situada hacia una educación intercultural e incluyente para y en la convivencia.

\section{ABSTRACT}

The conception of education has implications in the definition and management of educational policy as well as the dynamics of the 
school institution in defining its processes and the role of educational actors; in particular the social and political responsibility that falls on the teacher. The purpose of this dissertation is to document and analyze one's own teaching practice based on the accompaniment in the development of research projects that support the theses, in the particular case, of students of the Maestria en Educación Básica (MEB) with a specialty in Pedagogy of Difference and Interculturality in Unidad 096 CDMX Norte of the Universidad Pedagógica Nacional. Counseling is the accompaniment in the development of research projects, it considers one's own teaching practice as an object of knowledge and a link between academic work and classroom learning processes; this was documented in observation records. For the understanding of the educational fact, in its phenomenology through an interdisciplinary perspective, it allows to recover the discussion that the teachers carry out, initially, the conceptualization of the categories of analysis that explain the phenomena that current society lives and have implications in the school and, subsequently, to review and analyze the study plans, which are the basis for their implementation; in the orientation of the own teaching practice situated towards an intercultural and inclusive education for and in coexistence.

\section{PALABRAS CLAVE / KEY WORDS}

Educación Inclusiva; Educación Intercultural; Práctica Educativa; Enfoque Intercultural / Inclusive Education; Intercultural Education; Educational Practice; Intercultural Approach.

\footnotetext{
Y es en afirmar la "razón del Otro" que podemos empezar a identificar las semillas de pedagogías y praxis "otras", pedagogías y praxis radicales que parten de un deseo de "pensar con", que asumen la interculturalidad como proyecto-compromiso político, ético y epistémico, que buscan armar fuerzas decoloniales aliadas y que se dirigen hacia la construcción y movilización de poderes, seres, saberes, sociedades y mundos muy distintos. (Walsh, 2007, p. 34)
}

\section{INTRODUCCIÓN}

El proceso de conceptualización tiene el propósito de orientar el análisis para comprender la visibilización y el reduccionismo que el Estado hace de la diversidad cultural, esto permite evidenciar, no solo a las políticas culturales, de reconocimiento y educativas, sino, también, a la identidad nacional frente a los procesos de individuación y al sentido de pertenencia sociocultural.

La discusión, en el ámbito académico, mantiene vigente la crítica sobre las políticas para la 
inclusión y resemantización de la interculturalidad con el sustento del debate y la distinción entre el paradigma intercultural, el interculturalismo y la educación intercultural. En el caso de los docentes de educación básica, además de reflexionar y debatir estas nociones, es importante llevar a la práctica educativa el enfoque intercultural, en la perspectiva de una práctica situada en contextos multiculturales, mediante el desarrollo y gestión de procesos de aprendizaje y educativos hacia la convivencia.

El modelo de atención a la diversidad en la educación tiene implicaciones en la concepción, el diseño y la implementación del currículum; a su vez en la práctica educativa y los procesos que se desarrollan en el aula, de tal forma que resulta complejo armonizar con los propósitos para abatir la pobreza y las inequidades sociales.

Esta concepción implica un sesgo, respecto de la definición de la diversidad cultural y de la interculturalidad, pues en ella subyace la orientación hacia lo étnico (Dietz y Segura, 2006). Prevalece una visión antropológica sobre la interculturalidad basada en la concepción relativista de la cultura, que la reduce y soslaya la diversidad cultural. Algo similar sucede con la educación especial, más tarde la integración, y después, la inclusión educativa, estos últimos programas se implementaron para la atención de los estudiantes con alguna discapacidad (Crespo y Lalueza, 2003, Sagastizabal, 2004).

La convivencia, se concibe vinculada al eje de la convivencialidad: centrada en enseñar a resolver conflictos al enfrentar posiciones diferentes en la convivencia escolar; superar estereotipos, prejuicios, discriminación y prácticas pedagógicas encubiertas de racismo y dominación; así como valorar la diversidad cultural como un recurso para fortalecer las identidades y la pertenencia, está relacionada con el eje de equidad: asociada con el aspecto económico, señala que en el ámbito educativo se han reproducido relaciones de poder sobre minorías culturales en las cuales los indicadores educativos, tales como analfabetismo, ausentismo, reprobación, deserción y bajos niveles de aprendizaje se explican por las condiciones de vulnerabilidad, marginación, pobreza y exclusión. ( UNESCO, 2008)

La política de educación intercultural yel eje de la convivencialidad difieren diametralmente de los paradigmas de la interculturalidad y la convivencia establecidos por las políticas educativas debido a que, por principio porque dejan de lado el principio de desigualdad en el que se sustenta al sistema educativo y evaden las tensiones y contradicciones que genera al buscar reducirlo en el ámbito escolar; tiene sustento en la teoría del conflicto y en esta perspectiva la tarea es eliminarlo.

En el marco de las políticas educativas la educación ha sido adjetivada, como si en eso consistiera su valor, y lo que podemos observar es que conceptualmente, se vacía de contenido. La educación como factor de inclusión es objetivo de las políticas compensatorias y focalizadas, y es en esta perspectiva, que se le llena de adjetivos como una forma de respuesta a problemas estructurales y sociales que requieren respuesta.

Esta perspectiva está más orientada a la atención de la pobreza y a la generación de oportunidades en el marco de la escolarización. En tanto, la educación intercultural confiere la apreciación de la diversidad cultural, la relevancia y significación de los aprendizajes pero se aleja de los procesos de reconocimiento y alteridad.

La política de educación intercultural ha sido la respuesta para tratar de forma distinta a colectivos percibidos como diferentes con la finalidad de interculturalizar tanto el currículum 
como la práctica en un marco de tolerancia y reconocimiento con base en los principios de discriminación positiva e igualación de circunstancias de elección (Dietz y Segura, 2006) que aseguren la convivencialidad. Toda relación entre los individuos en sociedad genera disensos y tensiones, que, en el marco de la alteridad habrán de tener su curso.

El paradigma intercultural parte del respeto, disposición al diálogo, participación, negociación y cooperación, en la relación de sistemas culturales diferentes implica comprender la interdependencia de los actores sociales en el contexto de sus culturas, pueblos y grupos sociales a partir de marcos de referencia interdisciplinarios para una convivencia hacia la construcción del nosotros (Lenkersdorf, 2008).

\section{LA PRÁCTICA DOCENTE PROPIA EN SU CIRCUNSTANCIA}

La educación intercultural y la inclusión en el marco de las políticas de la justicia social, tiene su referente, en una perspectiva holística, con el concurso de diferentes concepciones frente al pensamiento único y universal; rupturas entre la concepción lineal y de continuidad de cara a las discontinuidades, entre la larga y la corta duración, con una postura abierta a nuevos horizontes.

En suma, la historia del pensamiento, de los conocimientos, de la filosofía, de la literatura parece multiplicar las rupturas y buscar todos los erizamientos de la discontinuidad; mientras que la historia propiamente dicha, la historia a secas, parece borrar, en provecho de las estructuras más firmes, la irrupción de los acontecimientos. (Foucault, 2002, p. 8)

La investigación tiene un carácter cualitativo, inicialmente; la problematización, se acompaña de un proceso de historización para enmarcar la indagación y el análisis del discurso de las políticas culturales y educativas para la inclusión con una mirada abarcadora. La comprensión del contexto multicultural precisa un posicionamiento desde el pensamiento complejo que permita identificar las discontinuidades y sea aprehendida en el marco de la interdisciplina, con el propósito de abordar el objeto de conocimiento en sus dimensiones y la conceptualización de las categorías de análisis para, desde un enfoque interpretativo, dar cuenta del estado de la cuestión de la interpretación de la educación intercultural y la inclusión en el marco de la política educativa.

No se trata de retomar la ambición del pensamiento simple de controlar o dominar lo real. Se trata de ejercitarse en un pensamiento capaz de tratar, de dialogar, de negociar, con lo real $[. .$.

Mientras que el pensamiento simplificador desintegra la complejidad de lo real, el pensamiento complejo integra lo más posible los modos simplificadores de pensar, pero rechaza las consecuencias mutilantes, reduccionistas, unidimensionales y finalmente cegadoras de una simplificación que se toma por reflejo de aquello que hubiere de real en la realidad. (Morin, 2003, p. 22)

La aproximación a la fenomenología de la práctica educativa es la vía para documentar el acompañamiento del trabajo docente de estudiantes de la Maestría en Educación Básica (MEB), de la especialidad Pedagogía de la Diferencia y la Interculturalidad, enfocado hacia las siguientes temáticas: 
Profesora 1. Procesos dialógicos: el aprendizaje como experiencia de identidad en alumnos de primaria

Profesora 2. La coeducación en el aula, una mirada desde los estereotipos de género.

Profesora 3. Educación Inclusiva en el aula.

Profesora 4. La ética como camino a la comprensión de la diferencia.

La sistematización del acompañamiento en el curso de las investigaciones que sustentan las tesis, se llevó a cabo, con el objetivo de una aproximación diagnóstica para comprender la práctica educativa e identificar, en su desarrollo, los procesos de alteridad en las interacciones sociales que se gestan en el ámbito educativo y los procesos escolares en el aula. Con la premisa de que la práctica docente es única, no se pretende realizar un estudio comparativo de las investigaciones motivo de análisis.

Para los fines de este diagnóstico se recupera la información derivada del acompañamiento en el desarrollo de las investigaciones, mediante un registro de observación, y la interpretación del mismo .

La asesoría de tesis en la MEB es un proceso de acompañamiento para el desarrollo de una investigación que tiene como objeto de conocimiento la práctica docente propia. El propósito es vincular el trabajo académico con los procesos de aprendizaje en el aula, en la perspectiva de las líneas de investigación de la Pedagogía de la Diferencia y la Interculturalidad, con una mirada abarcadora e interdisciplinar sobre el hecho educativo y la comprensión de la práctica docente, más allá del reduccionismo de la política educativa centrada en la Educación Intercultural y la Educación Inclusiva.

El registro de observación se conforma por categorías e indicadores que permiten dar cuenta de la implementación de las propuestas derivadas de las investigaciones, en la lógica establecida por cada una de éstas.

Durante las asesorías hubo espacio para discutir, desde diferentes posturas, la concepción de la práctica docente, la escuela, el enfoque intercultural y la educación inclusiva como sustento para el desarrollo de los procesos de aprendizaje en el aula.

La asesoría, la revisión de los avances y el registro de la información son tres momentos complementarios para documentar el acompañamiento y permiten una valoración de conjunto sobre la práctica docente situada, en la búsqueda de su comprensión y resignificación, circunscrita en un marco curricular establecido.

Es pertinente señalar que la última parte del acompañamiento se realizó en línea, debido a las condiciones que impone la pandemia.

\section{MARCO GENERAL}

Las investigaciones que desarrollan cuatro estudiantes de la MEB sustentan las propuestas temáticas arriba señaladas; cada investigación tiene una lógica propia, así como construcción teórica y metodológica definida. 
Las estudiantes son profesoras de educación básica en el nivel primaria, dos de ellas son maestras normalistas con quince años de experiencia; las otras dos son licenciadas en educación y pedagogía, con veinticinco y seis años de experiencia, respectivamente.

Es pertinente señalar que el ejercicio de la profesión docente, está relacionado con sus antecedentes formativos, la experiencia y el interés personal, a decir de las profesoras, fue determinante su orientación vocacional; esto lo refieren con gran emotividad y se manifiesta en el interés por realizar de mejor manera la práctica educativa.

Independientemente de su formación, las docentes expresan que sus referentes para la ejercicio del magisterio son: "como les enseñaron", y, si tuvieron una buena experiencia tratan de replicarla, pero, si la experiencia no fue positiva, se esfuerzan por no repetirla.

La trayectoria profesional en el ámbito de la educación es importante para la constitución de las instituciones, con base en los procesos de aprendizaje y la implementación de los proyectos educativos, porque esto permite recuperar la experiencia, valorar los cambios y fortalecer el sistema educativo nacional.

El ejercicio profesional de las maestras es en la educación pública, de la Secretaría de Educación Pública (SEP), de manera particular en la educación primaria, en sus diferentes grados. La función que desempeñan actualmente es de maestra frente a grupo. La antigüedad y, sobre todo, la experiencia las ha llevado a tomar la iniciativa de continuar su formación para mejorar su práctica docente y como factor de desarrollo personal.

Las problemáticas que identifican en el desarrollo de su práctica están relacionadas con la interacción entre los actores del proceso educativo en la escuela y el aula. No obstante, que los planes y programas de educación primaria marcan una clara tendencia a priorizar las asignaturas de Lenguaje y comunicación y Matemáticas (en la orientación del aprender a aprender), las maestras realizan una importante reflexión sobre las relaciones sociales y la convivencia en el aula y la escuela.

Este desplazamiento de la mirada marca un punto de inflexión hacia una de los propósitos fundamentales de la educación:

[...] la preocupación por la paz, la inclusión y la justicia social. Los principios éticos y morales de una visión humanista del desarrollo se oponen a la violencia, la intolerancia, la discriminación y la exclusión. [...] la educación y la instrucción, suponen dejar atrás el utilitarismo y el economicismo de cortas miras para integrar las dimensiones múltiples de la existencia humana. Esta visión hace hincapié en la inclusión de personas que frecuentemente son discriminadas: mujeres y niñas, poblaciones autóctonas, personas con discapacidades, migrantes, las personas mayores y las poblaciones de países afectados por un conflicto. Exige un planteamiento abierto y flexible del aprendizaje, que debe extenderse tanto a lo largo como a lo ancho de la vida: un planteamiento que brinde a todos la oportunidad de realizar su potencial con miras a un futuro sostenible y una existencia digna. (UNESCO, 2015, p. 10)

La consideración de las docentes, sobre la importancia de la interacción social en los procesos educativos, está encaminada a generar relaciones de respeto y ambientes propicios para el aprendizaje. En este orden de ideas, destaca la orientación de las temáticas de investigación ubicadas en el Área de formación académica: Exploración y comprensión del mundo material y social; y el campo de Formación personal y social: Educación 
socioemocional de los Planes y programas de estudio 2017, esta pauta resulta significativa para esta investigación porque refiere las interacciones sociales y la conviencia en los procesos educativos en el aula y la escuela,.

La definición de las temáticas también se explica por la atención y sensibilidad para identificar las problemáticas de mayor relevancia en su quehacer docente: la convivencia en la escuela y el aula.

\section{INVESTIGACIONES EN CURSO}

El proyecto de investigación que desarrollan las docentes se deriva del trabajo académico en el marco del programa de Maestría en Educación Básica (MEB). La definición de las temáticas de investigación, tienen su antecedente en la problematización para identificar los espacios de la práctica docente que eran necesarios de conocer para mejorar los procesos de aprendizaje.

Contrario al planteamiento discursivo de la política educativa las investigaciones se posicionan en la conceptualización sobre el sujeto en su historicidad, circunstancia y relaciones que establece en contextos multiculturales, de desigualdad y exclusión.

Posterior a la definición de su objeto de conocimiento y la elaboración del proyecto (aprobado por el asesor), de manera paralela, las maestras inician las gestiones para que las autoridades escolares les permitan realizar la investigación en la institución en la que trabajan, con el grupo que tienen asignado. Inicialmente, en el caso de las cuatro profesoras encontraron ciertas resistencias para la realización de la investigación por parte de las autoridades inmediatas y el cuestionamiento de sus pares. Finalmente, conseguir la autorización les genera certeza y confianza para el desarrollo de la pesquisa, junto con la seguridad que aporta al fortalecimiento de su formación respaldado por la formación en el posgrado.

En el marco del paradigma del aprendizaje, las profesoras asumen la responsabilidad de acompañar la construcción de aprendizajes, destacan la importancia del proceso formativo de la educación y la escuela, y esto lo ponderan con el entorno familiar y social en el que se desarrollan los alumnos. Esto determina la complejidad para el desarrollo de la investigación de carácter cualitativo, para dar cuenta las relaciones de los actores educativos en la escuela y el aula en la perspectiva de las temáticas definidas, esto implicó establecer categorías, variables e indicadores para la investigación in situ.

En el marco de las investigaciones en curso, las profesoras manifiestan que éste ha sido uno de los procesos más difíciles de la investigación, pero que la base conceptual para la categorización ha sido un elemento fundamental para el diagnóstico y la propuesta.

Sólo una de las investigaciones se planteó la necesidad de recabar información de los diferentes actores educativos de la escuela, con el propósito de identificar y documentar las problemáticas que se enfrentan de manera regular los docentes y su percepción sobre estos eventos. Inicialmente, la idea era aplicar una encuesta en línea, en dos escuelas, pero los profesores manifestaron franca oposición para colaborar. La encuesta se aplicó de manera física sólo en la escuela en la que labora la maestra y de veintinueve profesores entre docentes 
y autoridades, sólo respondieron el cuestionario quince. En general los instrumentos y técnicas para recabar información directa tuvieron buena acogida y permitieron disponer de información pertinente para el objetivo de cada investigación.

Para las cuatro tesistas, el conocimiento acompañado de la experiencia fueron determinantes para la definición de dimensiones, categorías, variables e indicadores de manera adecuada. La valoración de cada una de las investigaciones sugiere que el desarrollo de la actividad docente, en este marco, presenta constantes que se convierten en referentes permanentes, y, a su vez, en ejes problemáticos para guiar el análisis, vincular la teoría con la curriculares. Esto permitió una reflexión que pondere la información en forma general y, desde una perspectiva abarcadora, la comprensión de la práctica docente, para hacer la resignificación informada y contextualizada de la misma.

La información que aportaron los actores educativos, mediante el análisis, rebasó las respuestas a las preguntas iniciales; la información recabada fue basta y puede ser motivo de un análisis y reflexión más amplio.

Las profesoras, posterior a la investigación documental y la recuperación de la información directa, se posicionan en una situación de ventaja para realizar una práctica informada, pero sobre todo, con una clara intencionalidad. La categorización implicó una mejor comprensión de los referentes teóricos para realizar con pertinencia la implementación del currículo establecido. Mencionar esto último es de capital importancia, porque las cuatro profesoras desarrollaron su investigación tomando como punto de referencia los marcos curriculares vigentes para la elaboración de sus propuestas.

En la educación básica, sobre todo pública, existe un ordenamiento implacable con relación a guiar su práctica en observancia de los planes y programas vigentes, complementados con los libros de texto. Así que las profesoras reorganizaron sus planeaciones y tiempos establecidos para cada asignatura, con el propósito de realizar su investigación.

En este orden de ideas, con justicia Ángel Díaz Barriga señala:

Tantos temas cercanos a los alumnos que obviamente un sistema central no puede enfrentar, pero sí, se podría asignar a cada escuela, al colectivo docente, al Consejo Técnico planificar cómo trabajar con los niños de su escuela, que temas desarrollar, que preguntas focales realizar, ellos sí conocen diversas situaciones del entorno que rodea a los alumnos (Díaz-Barriga, 2021, s/p.).

Algunos elementos que no se documentan pero, que por la propia lógica de los programas encuentran y aprovechan, es que las competencias o en su caso, los llamados aprendizajes clave son procesuales e interdisciplinares, eso ha permitido a la intervención mediante proyectos o situaciones didácticas eficientar los tiempos, con relación al avance en contenidos programáticos.

Una ventaja de las docentes es que conocen a detalle los programas de estudio y en ese marco, han logrado colocar sus propuestas. Es decir, no están proponiendo contenidos nuevos o distintos; la diferencia se encuentra en el enfoque (intercultural) y el sustento (teórico y metodológico) de la investigación, que promueve la construcción de aprendizajes como base para el logro educativo. 
¿Qué descubrí? Que la verdadera historia de la educación está en las entrañas de las escuelas, en las vidas humanas, en la comunidad escolar, los maestros, los alumnos, los padres de familia, las autoridades escolares de carne y hueso, i.e., los supervisores, etc., y no en las autoridades educativas institucionales. En la institución, llámese SEP, autoridad educativa local, sindicatos, o supervisiones, se pierde la esencia de la educación y la sustancia del aprendizaje. (Andere, 2021, s/p.)

Las investigaciones recuperadas en este diagnóstico ofrecen elementos que ilustran el trabajo cotidiano de las maestras, ellas se apropian de la discursividad de los programas y en la mayor parte de los casos, de las ideas básicas; ofrecen una crítica informada sobre los mismos, pero también, y es el aspecto de mayor valía de estos procesos de investigación, es que ofrecen alternativas para su implementación.

En el análisis de las investigaciones, destacan fuertes contradicciones entre lo que plantea el discurso de la política educativa y los aprendizajes que deben construir los estudiantes, además de las indicaciones que reciben por parte de las diferentes autoridades. Esto, porque se combinan los planes y programas con los programas especiales y las reglas de operación sobre temáticas como interculturalidad, derechos humanos, personas con alguna discapacidad e inclusión.

Las docentes han expresado las dificultades para trabajar las asignaturas y los contenidos que las conforman con referencia a los programas anteriores, actualmente tienen que combinar, a saber:

1. En primero, segundo y tercero de preescolar; primero y segundo de primaria; y primero y segundo de secundaria se aplicará el plan de estudios 2017.

2. b) En tercero, cuarto, quinto y sexto de primaria; y en tercero de secundaria, se aplicará el plan de estudios 2011, y los componentes de "Autonomía curricular" y "Desarrollo personal y social" del plan de estudios 2017. (SEP, 2019, p. 184)

Además los contenidos se estructuran con temas cercanos a los alumnos que desde la generalidad no se pueden abordar, pero sí, se podría asignar a cada escuela, al colectivo docente, al Consejo Técnico la tarea de cómo trabajar con los niños de la escuela en sus aulas, en el sentido de temas focales a desarrollar, qué preguntas realizar, etc., para la comprensión del entorno y situaciones inmediatas.

Los retos que comparten las profesoras en sus investigaciones están relacionados con el cómo abordar la práctica docente desde el enfoque intercultural y la educación inclusiva, los Campos de Formación de los Plan y programas 2011; y, las Áreas de formación académica y los campos de desarrollo personal y social del Plan y programas 2017. Los programas sugieren cierta flexibilidad para la intervención del docente, sin embargo el enfoque es prescriptivo y reduccionista. La orientación predominante, es principalmente hacia el desarrollo de las competencias propias de las asignaturas y de las habilidades establecidas en el programa que conduzcan al logro de los aprendizajes esperados, relacionados con los contenidos más que con los procesos de aprendizaje.

Existe una variable sobre la perspectiva del conocimiento del programa y la implementación adecuada del mismo; esto coloca en el centro de la discusión la premisa 
sobre la distancia acerca del conocimiento, comprensión y manejo del programa por parte de las docentes, la cual, denota su apropiación del discurso. También exhibe la contradicción del enfoque que sustenta el aprendizaje en la educación básica y, por lo tanto, de estrategias, actividades, metodología, didáctica y formas de evaluación e incluso contenidos propios de los planes de estudio y las asignaturas; frente a la propuesta organizada, sustentada teórica y metodológicamente en las propuestas que incluyen las investigaciones referidas.

La educación intercultural y la educación inclusiva en congruencia con los principios de la política educativa están alineados con las políticas sociales compensatorias; esto marca no sólo una diferencia, sino también distancia del paradigma intercultural, la teoría del reconocimiento y la teoría de la acción comunicativa. La reflexión y el discernimiento para elaborar propuestas, sustentadas en el enfoque intercultural, que rebasen las políticas para la inclusión; requiere de un trabajo cuidadoso y metodológicamente pertinente para acceder a los aprendizajes, así como a las relaciones interculturales e incluyentes.

La educación básica remite a una concepción específica de educación y de aprendizaje; con el propósito de que los alumnos desarrollen competencias básicas para el logro de los aprendizajes esperados. Las investigaciones motivo de análisis plantean la mediación docente de manera clara, ésta queda explícita en la definición e implementación de los procesos de aprendizaje sustentados teórica y metodológicamente para el desarrollo de estrategias, proyectos y secuencias didácticas en la perspectiva del enfoque intercultural: sustentado en el diálogo de saberes.

\section{PUNTOS DE REFLEXIÓN}

El análisis de las investigaciones de las profesoras exhibe la importancia de la comprensión de la práctica educativa como un proceso dialógico entre los actores educativos; y, entre los sujetos con el conocimiento. En esta perspectiva, la planeación va más allá de las actividades escolares que realizan las maestras, es una organización del proceso educativo que implica una postura, además del trabajo que "no se ve" y el que se realiza fuera de horario.

Asimismo, las respuestas de los maestros en la encuesta y las entrevistas manifiestan el interés y la necesidad del trabajo colegiado. Un análisis inferencial, permite observar que, por sus propios medios y, con cierta regularidad, conducen a los alumnos hacia la sistematización de los contenidos de aprendizaje mediante el desarrollo de actividades de análisis, reflexión y síntesis, con base en las orientaciones de los planes y programas y la información de los libros de texto, sin reparar en el enfoque teórico o las metodologías que pautan el ejercicio docente.

El uso y abuso del libro de texto, es una constante: La ausencia del libro de texto en los procesos de enseñanza puede ser dramática, porque para algunos profesores o en algunos contextos es "el recurso" por excelencia. Y siguen a pie juntillas las recomendaciones por ser la única opción para solventar el abordaje de los contenidos de la asignatura y las actividades correspondientes.

Sin embargo, para las temáticas que orientan las investigaciones que se analizan en esta aproximación, el libro de texto puede ser un elemento que se complemente con otros recursos y actividades de aprendizaje, pues el objetivo de las investigaciones se ocupa del 
desarrollo de procesos que se viven en la interacción social. Esta situación toma distancia de la práctica tradicional porque se requiere poner en juego las habilidades sociales para la convivencia.

Las docentes identifican necesidades sobre la práctica y la conformación del colectivo docente. Todas coinciden en que los maestros deben reflexionar en y sobre la práctica docente, sistematizar esa reflexión y preferentemente, acompañar la práctica docente con la investigación, además de realizar trabajo colegiado y generar un espacio para compartir saberes y experiencias.

Las profesoras sugieren que los maestros en general necesitan desarrollar habilidades de investigación como: la búsqueda de información, conocer la temática, arribar a procesos de análisis y argumentación, para documentar la práctica propia: su experiencia y sus saberes. El modelo de comunidades de indagación propone el aprendizaje a través de la creación de comunidades para construir el conocimiento a partir de intereses, inquietudes y experiencias comunes con el propósito de generar la reflexión individual; el conocimiento se construye a partir de comunidades pero se propicia la expresión de la individualidad de los integrantes y la reflexión de cada uno de ellos hacia el conocimiento: social, cognitivo y docente (Anderson. Rourke, Garrison y Archer, 2001).

En el contexto de la pandemia y las condiciones de confinamiento el uso de los medios de comunicación y la tecnología para apoyar los procesos de aprendizaje en la educación, se ha extendido de manera significativa, pero no con suficiencia en términos de disposición de dispositivos, conectividad, conocimientos y habilidades digitales. Hoy, la educación remota parece haber llegado para quedarse, con las implicaciones que conlleva al acentuar las desigualdades en el acceso, la polarización social y la desconexión como una nueva forma de exclusión.

De primera intención, la idea fue pasar la escuela vía remota, no se consideró elaborar programas apropiados para la educación en línea. En el marco de una situación excepcional, se mantuvo la exigencia de dar continuidad a la implementación de los programas para "evitar el rezago" y que se "perdieran" clases e incluso el ciclo escolar. Esto ha generado un abuso hacia los docentes quienes deben estar disponibles todos los días a todas horas para las autoridades, padres de familia y estudiantes; la carga burocrática ha incrementado y se suman cada vez más responsabilidades para los maestros, debido a la falta de regulación del trabajo en casa.

Los cambios socioculturales, el desarrollo acelerado de las tecnologías y la sociedad de la información plantean desafíos para la educación y los procesos de aprendizaje. La educación, la institución escolar y los diversos actores educativos tendrán que fortalecer la educación mediante la incorporación de las habilidades digitales y las Tecnologías de la Información y la Comunicación (TIC). (UNESCO, 2015)

La educación vía remota muestra una tendencia en convertirse en una actividad que acompañará a la educación de forma complementaria o combinada, esto porque el presupuesto ha disminuido drásticamente y el escenario post pandemia se dibuja complicado en términos de infraestructura y financiamiento.

La educación tiene que encontrar los medios de responder a estos desafíos, tomando en consideración las numerosas cosmovisiones y los sistemas de conocimiento alternativos, 
así como nuevas fronteras de la ciencia y la tecnología, por ejemplo, los avances de las neurociencias y las novedades de la tecnología digital. Nunca ha sido más urgente replantear la finalidad de la educación y la organización del aprendizaje. (UNESCO, 2015, p. 8)

Los retos que se avecinan son mayúsculos. Justamente los sectores de la población excluidos: los pueblos indígenas, las personas con alguna discapacidad, además de los pobres y extremadamente pobres, quedaron al margen de la educación remota con el Aprende en casa.

\section{CONCLUSIONES}

La educación es un proceso humano y, a su vez, un proceso eminentemente social; por lo tanto como un hecho social, para definirla desde una perspectiva social, los especialistas plantean de manera general, dos concepciones: una como transmisión y otra como transformación. Las profesoras autores de las investigaciones que se incluyen en este diagnóstico, optaron por concebir a la educación como transformación y en esa perpsectiva asumieron el enfoque intercultural, que de suyo es holístico e incluyente.

La inclusión como política de estado ha promovido mayor visibilidad de los diferentes y políticas más puntuales, mediante el modelo de atención a la diversidad en la educación, pero son insuficientes y poco pertinentes. "Las instituciones y las políticas educativas están atrapadas en un océano de normas, intereses y centralizaciones que le son imposibles de superar" (Andere, 2021 s/p.). Sin embargo, la diferenciación y segmentación sociales y la definición de la diversidad cultural de manera fragmentada van a contracorriente del enfoque intercultural.

Es en este contexto que la NEM demanda de: "La tarea docente demanda tiempo para pensar y diseñar las mejores situaciones de aprendizaje o actividades didácticas, así como para evaluar los logros de los estudiantes" (SEP, 2019, p. 187).

La atención de la diversidad sociocultural tiene implicaciones en el diseño, concepción e implementación del currículum, la práctica educativa y los procesos que se desarrollan en el aula, de tal forma que resulta complejo armonizar con los propósitos para abatir la pobreza y las inequidades sociales. El enfoque sobre la educación intercultural que adoptan las políticas sociales y en consecuencia las políticas culturales y educativas marcan una tendencia hacia la inversión en capital humano:

Es por esa razón que nuestra Estrategia de Educación 2020 tiene como meta lograr el Aprendizaje para Todos. El Aprendizaje para Todos implica garantizar que todos los niños y jóvenes -no únicamente los más privilegiados o los más dotados- puedan no solo asistir a la escuela, sino también adquirir los conocimientos y las capacidades que necesitan para llevar vidas saludables y productivas y obtener un empleo significativo. Los tres pilares de nuestra estrategia son: Invertir temprano. Invertir con inteligencia. Invertir para todos. (BM, 2011, p. 4)

Los organismos internacionales, como entidades que marcan la agenda de las políticas sociales, con cierta frecuencia renuevan su compromiso con el vínculo de la educación con el ámbito económico, en particular el aparato productivo. En consecuencia, las metas en la educación se plantean hacia la adquisición de conocimientos y el desarrollo de capacidades 
para que los individuos se inserten al aparato productivo mediante el empleo.

Esta perspectiva sobre el sistema educativo y la educación escolarizada representa una presión importante para los docentes; en tanto deben cumplir con un programa que establece prioridades de manera pragmática; frente a los procesos en el cotidiano de la institución escolar, que van más allá del aprender a aprender.

La relación de la sociedad con el Estado, va de la mano con la construcción de una sola y única visión del mundo, producto de un orden jerarquizado, selecciona un repertorio específico de elementos para generar un orden social, un esquema racional para la sociedad y a nivel internacional se hace lo propio para la humanidad entera, elaborando mecanismos para asegurar la continuidad, basada en el poder de unos y la subordinación de otros

La práctica educativa hoy tiene una responsabilidad social y política que recae en el docente. La discusión que los docentes están obligados a dar, primero mediante la conceptualización de las categorías de análisis que explican los fenómenos que vive la sociedad actual y tienen implicaciones en la escuela, posteriormente, hacer la revisión y análisis de los planes de estudio, que les permita hacer una implementación de los mismos, situada y en la perspectiva de una educación intercultural e incluyente para y en la convivencia.

En las investigaciones referidas, las profesoras hacen una reconocimiento de la práctica propia en el contexto normativo y el entorno escolar; con base en su experiencia, sus saberes y conocimientos establecen rutas de ruptura con la modelización de la práctica educativa y configurar procesos atingentes a las problemáticas que se presentan en su quehacer cotidiano y ofrecer respuestas pertinentes y sustentadas en el enfoque intercultural.

Con la premisa de que la desigualdad y la diferencia propician discriminación y exclusión de diversos grupos sociales las profesoras identifican "problemas de gran magnitud" en la escuela y el aula relacionados con base en este supuesto, se dan a la tarea de discutir el concepto de educación y su finalidad:

PROFESORA 1: “Tenemos esperanza en que todo cambiará y mejorará.Teóricamente este cambio tendría que darse en la educación, ya que es el punto social en donde todos los integrantes de una sociedad interceptamos una idea común: mejorar la sociedad a través de la educación".

PROFESORA 2: “La modificación de las prácticas y el concepto de educación tiene que impactar las relaciones en los procesos de aprendizaje en el aula".

PROFESORA 3: “La educación está relacionada con la valoración del proceso educativo con relación al sujeto".

PROFESORA 4: “La educación, es la manera más natural de adentrarse al conocimiento pero las políticas públicas se enfocan en ampliar la cobertura como sinónimo de lograr una educación equitativa".

Estasreflexionesseconviertenenejes problematizadores, generadoresdecuestionamientos para conocer y comprender el hecho educativo desde la mirada de las investigadoras. En este proceso hay planteamientos de búsqueda, conocimiento, conceptualización, pero sobre todo de comprensión y significación, que son la base para la crítica de los marcos normativos que determinan la práctica docente y que permiten la resignificación de la misma. Posicionar 
al sujeto en el centro de la educación tiene como consecuencia, establecer que si bien la educación es una tarea de Estado, también es un asunto de interés e impacto social. Cuando se habla de cambio, mejora e impacto las docentes se refieren, al mediano y cortos plazos, en el sentido de que, inicialmente los alumnos manifiestan evidencias de ello y así sucede, pero el cambio, la mejora o la transformación se generan en la práctica.

PROFESORA 1: "La convivencia escolar como el conjunto de prácticas relacionales de los agentes que participan de la vida cotidiana de las instituciones educativas, constituyen un elemento sustancial de la experiencia educativa, en tanto que la cualifican. Estas prácticas relacionales, -observables en los procesos de enseñanza, en el manejo de normas, en la construcción de acuerdos, en la solución de conflictos, en la evaluación, en el reconocimiento de las diferencias, en el trato con los padres, en las interacciones entre los estudiantes, y con sus docentes dan lugar a procesos de inclusión o de exclusión, de participación o segregación, de resolución pacífica o violenta de las diferencias, entre otros.

PROFESORA 2: "Modificar las prácticas estereotipadas en el aula y en la escuela para que no encuentren refuerzo en la familia y en la sociedad".

PROFESORA 3: “Conocer y comprender la práctica para poder cambiar”.

PROFESORA 4: “Un proceso dialógico posibilita una educación intercultural donde se comprendan las diferencias, como un valor y no como desigualdad. Esto manifiestan los alumnos que han mejorado su autoestima al sentirse parte del grupo y han incrementado sus habilidades interpersonales como: reciprocidad, empatía, colaboración y negociación".

El enfoque intercultural se construye en el marco de los objetos de estudio y las profesoras -investigadoras lo llevan a la práctica.

En consecuencia los sujetos que participan hacen de la práctica un momento constitutivo para establecer la relación pedagógica hacia la interculturalidad en el plano de la ética, los procesos dialógicos, la perspectiva de género y la educación inclusiva.

Profesora 1: "La interculturalidad se debe establecer como un enfoque que hace que entendamos, comprendamos y dejemos ser al otro. Diferentes, somos todos".

PROFESORA 2: “La condición de igualdad en la perspectiva de género, nos recuerda que la condición de igualdad es humana".

PROFESORA 3: "La inclusión considera la planeación por cada niño con alguna discapacidad para darle un trato diferenciado y con el propósito de que aprendan lo mismo que los alumnos regulares ¿La inclusión consiste en el logro de aprendizajes? “

PROFESORA 4: “Desde la perspectiva intercultural se propone una reconceptualización del valor de la diferencia hacia principios de igualdad, justicia y libertad. Ello conlleva aceptar la cultura escolar como diversidad y reconocer que la escuela se convierte en una comunidad de aprendizaje permanente".

Todos los sujetos de la educación (y no solo las personas con capacidades especiales y discapacidades) tenemos biología y necesitamos comportarnos en relación con un entorno que nos constituye y que a la vez contribuimos a cons- truir, en una relación dialógica de co-construcción. (Bacigalupe, 2020, p. 238-239) 
El valor pedagógico del enfoque intercultural en la educación está en los procesos diálogicos en:

La producción de conocimientos, el diálogo de saberes; el proceso de mediación docente para el aprendizaje.

La acción comunicativa en la que expresa la identidad y el sentido de pertenencia.

La perspectiva de género en la consideración de condiciones de igualdad.

La ética como práctica en la conjunción de leyes y virtudes; inteligencia y voluntad; imaginación y sensibilidad en la interacción social.

La integración escolar no es sinónimo de inclusión. La inclusión está basada en la consideración de que todos somos iguales y diferentes; y, las relaciones se gestan sobre una base de reconocimiento, respeto y comunicación para y en la convivencialidad.

Solo cuando se reconocen las tensiones y las contradicciones el antagonismo es posible, para pensar y reconocer la existencia de la alteridad que no puede tener una "solución racional", conlleva a la exploración de respuestas y decisiones que requieren una alternativa. Se involucran identidades individuales y colectivas en la diferencia hacia la construcción de un nosotros y un ellos; y, no necesariamente alcanzar un consenso. En los procesos dialógicos se involucran tensiones y disensos y con base en el respeto a la diferencias para la conformación de nosotros.

La función del docente en tanto que acompaña los aprendizajes es acorde con las exigencias formativas establecidas por los marcos normativos que regulan el ejercicio de la docencia. en los planes y programas de estudio correspondientes. Esto resulta significativo para propiciar un aprendizaje centrado en la reflexión y el análisis.

[...] la interculturalidad apunta para la construcción de sociedades que asuman las diferencias como constitutivas de la demo- cracia y sean capaces de construir relaciones nuevas, verdaderamente igualitarias entre los diferentes grupos socioculturales, lo que supone empoderar aquellos que fueron históricamente considerados inferiores. (Walsh, p. 152).

El enfoque intercultural en la educación tiene impacto en los procesos y las prácticas, porque se basa en una concepción diferente de la realidad; no es un dogma y tampoco un modelo, ponen en tensión y cuestionamiento las estructuras. Precisa de la historización para la comprensión de la práctica situada y coloca en el centro a los sujetos y sus relaciones.

\section{REFERENCIAS}

Andere, E. (2021) Mi decepción con la política educativa: 1921-2021, en Educación Futura, 11 de enero 2021. Disponible en https://tinyurl.com/9z2478bbConsultado el 11 de enero de 2021.

Anderson, T., Rourke, L., Garrison, D.R. \& Archer, W. (2001). Assessing Teaching Presence in a Computer Conferencing Context. Journal of Asynchronous Learning Networks, 5 
(2), 1-17

Bacigalupe, M. A. Los estudios del comportamiento humanos en la construcción de la neurociencia educacional, en Interdisciplina 8, $\mathrm{n}^{\circ} 22$ (septiembre-diciembre 2020, pp. 223-245.

Banco Mundial (2011) Aprendizaje para todos. Invertir en los conocimientos y las capacidades de las personas para fomentar e desarrollo. Washinton D. C.: Banco Internacional de Reconstrucción y Fomento/Banco Mundial. Disponble en : https:// tinyurl.com/2sb8jd24

Consultado el 10 de enero de 2020.

Crespo, Isabel y Lalueza, José Luis. (2003) Culturas minoritarias, educación y comunidad en Essomba, M. A. (Ed.) Educación e inclusión social de inmigrados y minorías. Tejer redes de sentido compartido. Barcelona: Ed. Praxis.

Díaz-Barriga, A. (2021) Con relación a los resultados de una encuesta que presenta la SEP sobre de Aprende en casa 2, en Educación futura. 2 de enero de 2021. Disponible en https://tinyurl.com/3kbrawfw

Dietz, G., Segura, S. (2006). Multiculturalismo e interculturalidad, en Muñoz, H. Lenguas y educación en fenómenos multiculturales. México, UAM-Iztapalapa, Departamento de Filosofía/UPN.

Foucault, M. (2002) Arqueología del saber. Buenos Aires: Siglo XXI Editores.

Lenkersdorf, C. (2008). Aprender a escuchar. Enseñanza maya tojolabales. México: Plaza y Valdés Editores.

Morin, E. (2003) Introducción al pensamiento complejo. España: Ed. Gedisa.

Sagastizabal, M. A. (2004). Diversidad cultural y fracaso escolar. Educación intercultural: de la teoría a la práctica. Buenos Aires: Ediciones Novedades Educativas.

SEP (2019) Modelo educativo: Nueva escuela mexicana. México: Subsecretaría de Educación Básica.

UNESCO, (2008) Educación y diversidad cultural en la escuela. Lecciones desde la práctica educativa. Santiago de Chile, UNESCO, OREALC/ UNESCO Santiago.

UNESCO (2015). Replantear la educación. París: UNESCO.

Walsh, C. (2007). Interculturalidad, colonialidad y educación. Revista Educación y Pedagogía XIX(48), pp. 25-35. https://www.flacsoandes.edu.ec/sites/default/files/ agora/files/1265909654.interculturalidad_colonialidad_y_educacion_O.pdf

Walsh, C. (2003). Pedagogías decoloniales. Prácticas insurgentes de resistir, (re)existir y (re)vivir. Quito: Abya Yala. 